\title{
Cryptotanshinone exhibits therapeutical effects on cerebral stroke through the PI3K/AKT-eNOS signaling pathway
}

\author{
WEIXIN ZHU, WEIHONG QIU and AILAN LU \\ Department of Rehabilitation, Jinhua Municipal Central Hospital, Jinhua, Zhejiang 321001, P.R. China
}

Received June 30, 2016; Accepted July 13, 2017

DOI: $10.3892 / \mathrm{mmr} .2017 .7824$

\begin{abstract}
Cerebral stroke is a kind of acute cerebrovascular disease with high incidence, morbidity and disability. Treatments against various types of cerebral stroke are limited at preventive measurements due to the lack of effective therapeutic method. The present study aimed to investigate the protective effect of cryptotanshinone (CPT) on cerebral stroke, and investigate the possible mechanism involved in order to develop a novel therapy against stoke. The phosphoinositide 3-kinase membrane translocation of cerebral stroke rats pretreated with CPT at various concentrations were measured, as well as the phosphorylation of protein kinase B (AKT) and endothelial nitric oxide synthase (eNOS). Additionally, the expression level of B-cell lymphoma 2 (Bcl-2), Bcl-2-associated X protein (Bax) and vascular endothelial growth factor were also assessed using western blotting and reverse transcription-quantitative polymerase chain reaction. Furthermore, biochemical tests were used to measure the activity of superoxide dismutase (SOD), malondialdehyde (MDA) and nitric oxide (NO) in both the cerebral cortex and peripheral blood. As a result, CPT-pretreated rats presented declined phosphoinositide 3-kinase (PI3K) and AKT expression levels, indicating that the PI3K/AKT signaling pathway was inhibited. Increased Bcl-2 and NO levels in both the cerebral cortex and peripheral blood demonstrated the anti-apoptosis and blood vessel protection effect of CPT. Furthermore, increased SOD activity and declined MDA levels demonstrated suppressed lipid peroxidation. In conclusion, CPT exhibited a protective effect against cerebral stroke through inhibition of the PI3K/AKT-eNOS signaling pathway. These results suggested the potential of CPT as a promising agent in the treatment of cerebral stroke.
\end{abstract}

Correspondence to: Miss Ailan Lu, Department of Rehabilitation, Jinhua Municipal Central Hospital, 365 Moon Street, Jinhua, Zhejiang 321001, P.R. China

E-mail: ailanlu0626@163.com

Key words: cerebral stroke, cryptotanshinone, phosphoinositide 3-kinase/protein kinase B-endothelial nitric oxide synthase

\section{Introduction}

Cerebral stroke, also known as cerebral vascular accident (CVA), is a kind of acute cerebrovascular disease (1). Cerebral stroke, including ischemic stroke and hemorrhagic stroke caused by the blood vessels burst or vascular obstruction, results in brain tissue damage (2). Cerebral stroke has the characteristics of high incidence, morbidity and disability (3). Ischemic stroke has a higher incidence at $60-70 \%$ of the total number of cerebral apoplexy, while hemorrhagic stroke caused by the occlusion or stenosis of internal carotid artery or vertebral artery has a higher mortality in individuals aged 40 or older (4). At present, treatments against various types of cerebral stroke are mainly preventive due to the lack of effective therapeutic method.

Cryptotanshinone (CPT) extracted from Danshen (Salvia miltiorrhiza Bunge) has been reported to possess multiple pharmacological activities, including anti-cancer, anti-inflammatory, anti-oxidative, anti-diabetic and anti-obesity functions (5-9). Danshen, as a well-known traditional Chinese herb, is frequently used in the clinic in Chinese medicine to protect against several diseases, including cardiovascular diseases, diabetes, hepatitis, ischemic stroke, chronic renal failure and menstrual disorders. CPT isolated from Danshen is one of the major representative components (7). It has been reported to have obvious antitumor activity in a variety of cancer cells, including prostate carcinoma, hepatocarcinoma, rhabdomyosarcoma and melanoma cells $(10,11)$. Additionally, CPT was demonstrated to inhibit MCF7 cell proliferation by suppressing mechanistic target of rapamycin-mediated CyclinD1 expression and $\mathrm{Rb}$ phosphorylation that lead to MCF7 cell apoptosis by inducing stress in the endoplasmic reticulum (ER) in breast carcinoma cells (12). This suggested that CPT may be effective for the treatment of cerebral stroke, which has previously rarely been reported. Therefore, the present study aimed to explore the feasibility of CPT as an anti-cerebral stroke agent and investigate the possible mechanisms involved.

\section{Materials and methods}

Animal grouping and drug delivery. Male Sprague-Dawley rats (weight, 140-160 g; $n=36$ ) of specific pathogen free class were purchased from SLAC animal, Shanghai, China. Animals were kept with the maintenance of environmental conditions at 
$20-26^{\circ} \mathrm{C}, 40-70 \%$ and a 12 -h light/dark cycle, with free access to food and water. Rats were randomly allocated into 6 groups ( $n=6 /$ group): Blank control (rats underwent a sham procedure without model induction or treatment), control (cerebral stroke rats without the treatment of CPT), low dose CPT $(5 \mathrm{mg} / \mathrm{kg})$, medium dose CPT (15 mg/kg), high dose CPT (45 mg/kg) and LY294002 (Merck KGaA, Darmstadt, Germany). Rats in the CPT groups were treated with different concentrations of CPT (Sigma-Aldrich; Merck KGaA) through intragastric administration for $24 \mathrm{~h}$ prior to surgery. All animal study protocols were approved by the Institutional Animal Care and Use Committee of Zhejiang Province Center Hospital (Zhejiang, China; IACUC-20130315-01).

Cerebral stroke model in rats. Rats were treated with CPT for $24 \mathrm{~h}$, during which rats were fasted for the last $12 \mathrm{~h}$ of CPT treatment. A suture method was employed to generate a cerebral stroke model. Chloral hydrate (10\%; $0.35 \mathrm{ml} / 100 \mathrm{~g}$; Aladdin, Shanghai, China) was injected through intraperitoneal administration. Fully anesthetized rats were fixed on a plate at supine position and disinfected with iodine. The right side of subcutaneous tissue and gland were separated through the median incision on the neck of rats. The common carotid artery was ligated at the proximal part and the external carotid artery was ligated at the distal part. The internal carotid was blocked with a vascular clamp at the proximal part. An occlusion line was inserted into the internal carotid along the common carotid artery $\sim 18 \mathrm{~mm}$ until the sensation of resistance. The crotch of common carotid artery, external carotid artery and internal carotid was ligated so as to fix the occlusion line before the suture of neck incision.

Biochemical analyses. Measurements of superoxide dismutase (SOD), malondialdehyde (MDA) and nitric oxide (NO) levels in the cerebral cortex and peripheral blood were assessed using commercial kits [Nanjing Jiancheng Bioengineering Institute, Nanjing, China; cat. nos. A001-2 (SOD), A003 (MDA) and A012 (NO)]. Tissue samples homogenized in $100 \mathrm{mmol} / 1$ Tris- $\mathrm{HCl}$ buffer were centrifuged at $10,000 \mathrm{x}$ g for $20 \mathrm{~min}$ at $4^{\circ} \mathrm{C}$. The content of SOD, MDA and NO were determined and calculated according to the manufacturer's protocol.

Western blot assay. Cerebral cortex samples (20 mg) were cut into pieces and mixed with $250 \mu \mathrm{l}$ histiocyte lysis buffer (radioimmunoprecipitation assay buffer; Beijing Solarbio Science \& Technology Co., Ltd., Beijing, China) containing $0.01 \%$ protease inhibitor cocktail (Sigma-Aldrich; Merck KGaA). After fully lysing, samples were centrifuged at $12,000 \mathrm{x} \mathrm{g}$ for $15 \mathrm{~min}$ at $4^{\circ} \mathrm{C}$ and the supernatant was collected. A Bicinchoninic Acid protein quantification kit (Thermo Fisher Scientific, Inc., Waltham, MA, USA) was used to quantify the protein contents. Tissue samples $(15 \mu \mathrm{g})$ were separated by $10 \%$ SDS-PAGE and subsequently transferred to a nitrocellulose filter membrane (EMD Millipore, Billerica, MD, USA) electrophoretically. Blots were blocked with 5\% skimmed milk at room temperature for $1 \mathrm{~h}$, followed by incubation with anti-phosphoinositide 3-kinase (PI3K; cat. no. ab189403; 1:1,000; Abcam, Cambridge, MA, USA), anti-phosphorylated (p)-PI3K (cat. no. ab182651; 1:1,000; Abcam), anti-protein kinase B (AKT; cat. no. \#9272; 1:1,000; Cell Signaling
Technology, Inc., Danvers, MA, USA), anti-p-AKT (cat. no. \#9271; 1:1,000; Cell Signaling Technology, Inc.), endothelial nitric oxide synthase (eNOS; cat. no. \#32027; 1:1,000; Cell Signaling Technology, Inc.), anti-p-eNOS (cat. no. ab195944; 1:500; Abcam), anti-B-cell lymphoma 2 (Bcl2; cat. no. sc-492; 1:400; Santa Cruz Biotechnology, Inc., Dallas, TX, USA), anti-Bcl-2 X-associated protein (Bax; cat. no. sc-493; 1:400; Santa Cruz Biotechnology, Inc.), anti-vascular endothelial growth factor (VEGF; cat. no. ab46154; 1:1,000; Abcam) and anti-GAPDH (cat. no. \#5174; 1:1,500; Cell Signaling Technology, Inc.) primary antibodies at $4^{\circ} \mathrm{C}$ overnight, and then incubated with horseradish peroxidase-conjugated secondary antibodies at room temperature for $1 \mathrm{~h}$ [goat anti-mouse (cat. no. A0216) or anti-rabbit (cat. no. A0208) secondary antibody; 1:1,000; Beyotime Institute of Biotechnology, Shanghai, China]. Enhanced chemiluminescence (Thermo Fisher Scientific Inc.) was used to detect the blots visually.

\section{Reverse transcription-quantitative polymerase chain reaction} (RT-qPCR). Total RNA was extracted from tissue samples and quantified using TRIzol Reagent (Invitrogen; Thermo Fisher Scientific, Inc.). A reverse transcription kit (Fermentas; Thermo Fisher Scientific, Inc.) was used to synthesis cDNA through a total volume of $25 \mu \mathrm{l}(12 \mu \mathrm{l}$ RNA-primer Mix, $5 \mu \mathrm{l}$ 5X RT reaction buffer, $1 \mu 125 \mathrm{mM}$ dNTPs, $1 \mu 125 \mathrm{U} / \mu 1$ RNase inhibitor, $1 \mu 1200 \mathrm{U} / \mu \mathrm{l}$ M-MLV Rtase, $1 \mu \mathrm{l}$ Oligo(dt) $)_{18}$ and $4 \mu \mathrm{l}$ DNase-free $\mathrm{ddH}_{2} \mathrm{O}$ ). cDNA was then amplified using a SYBR Green PCR kit (Thermo Fisher Scientific, Inc.) through a total PCR system of $25 \mu \mathrm{l}$ (12.5 $\mu \mathrm{l}$ SYBR Green Mix, $0.5 \mu \mathrm{l}$

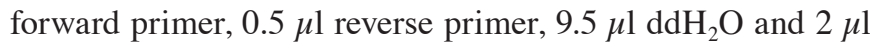
cDNA template). The PCR conditions consisted of initial denaturation for $10 \mathrm{~min}$ at $95^{\circ} \mathrm{C}$, denaturing for $15 \mathrm{sec}$ at $95^{\circ} \mathrm{C}$ and annealing for $45 \mathrm{sec}$ at $60^{\circ} \mathrm{C}$ for 40 cycles. Amplification kinetic curves were obtained for $15 \mathrm{sec}$ at $95^{\circ} \mathrm{C}, 1 \mathrm{~min}$ at $60^{\circ} \mathrm{C}$, $15 \mathrm{sec}$ at $95^{\circ} \mathrm{C}$ and $15 \mathrm{sec}$ at $60^{\circ} \mathrm{C}$. Primers used for the amplification are listed in Table I. Quantification of gene expression was performed using the $2^{-\Delta \Delta \mathrm{Cq}}$ method (13).

Statistical analysis. Data were analyzed by one-way analysis of variance followed by Tukey's post hoc test, using GraphPad Prism 6.0 software (GraphPad, La Jolla, CA, USA). Data are presented as the mean \pm standard deviation. $\mathrm{P}<0.05$ was considered to indicate a statistically significant difference.

\section{Results}

CPT inhibits the PI3K/AKT signaling pathway. The membrane translocation of PI3K and the phosphorylation of AKT during stroke were measured by using western blotting. As presented in Fig. 1, the phosphorylation levels of PI3K were markedly increased in the stroke group compared with the blank control. Additionally, cerebral stroke was able to induce significant phosphorylation of the PI3K downstream effector protein AKT ( $\mathrm{n}=3$ independent repeats; $\mathrm{P}<0.001$ ), and both PI3K membrane translocation and AKT phosphorylation were significantly attenuated in CPT groups. As a positive control, PI3K membrane translocation in the LY294002 group demonstrated a similar result compared with the CPT high dose group. Although there was significant difference in AKT phosphorylation between CPT high dose group and LY294002 
Table I. Primers used for reverse transcription-quantitative polymerase chain reaction.

\begin{tabular}{llll}
\hline Gene & \multicolumn{1}{c}{ Primer sequence } & Species & Amplicon size (bp) \\
\hline Bcl-2 & F: 5' GGGACGCGAAGTGCTATTGG 3' & Rattus norvegicus & 214 \\
& R: 5' GTTGCTCTCAGGCTGGAAGG 3' & & 188 \\
VEGF & F: 5' GAGTCTGTGCTCTGGGATTTG 3' & Human & 134 \\
Bax & R: 5' TCCTGCTACCTCTTTCCTCTG 3' & \\
GAPDH & F: 5' TTGACGGTAAGGACGGACTC 3' & Human & 181 \\
& R: 5' GGCGTTCCCATACTTCACAC 3' & Human & \\
\hline
\end{tabular}

F, forward; R, reverse; Bcl-2, B cell lymphoma 2; VEGF, vascular endothelial growth factor; Bax, Bcl-2-associated X protein.

group $(\mathrm{n}=3, \mathrm{P}<0.01)$, it demonstrated a tendency to decrease in the CPT groups dose-dependently. Furthermore, the expression level of endothelial nitric oxide synthase (eNOS) was measured. The eNOS expression was increased and attenuated in the stroke and CPT groups, respectively, in comparison with the control (Fig. 1).

CPT regulates downstream effector proteins. Furthermore, the expression level of VEGF was measured by using western blotting and RT-qPCR. The protein expression of VEGF activated by the PI3K signaling pathway was significantly increased in stroke rats compared with the blank control. However, the increased VEGF expression was attenuated by CPT in a dose-dependent manner (Fig. 2A and B). The expression levels of the AKT downstream proteins $\mathrm{Bcl} 2$ and $\mathrm{Bax}$ were also measured. $\mathrm{Bcl} 2$ protein expression levels decreased significantly in the stroke compared with the blank control $(\mathrm{n}=3, \mathrm{P}<0.001)$. However, decreased $\mathrm{Bcl} 2$ expression was attenuated in the CPT groups in a dose-dependent manner. On the other hand, Bax was markedly upregulated in the stroke group compared with the control $(n=3, P<0.001)$. Similarly, the increased expression level of Bax was attenuated by CPT dose-dependently. Both Bcl2 and Bax expression levels in higher dose CPT groups were restored to a more similar level to the LY294002 group, though there were significant differences $(\mathrm{n}=3 ; \mathrm{P}<0.05, \mathrm{Bcl} 2$ vs. LY294002; $\mathrm{P}<0.001$, Bax vs. LY294002).

CPT regulates $N O$ level. As presented in Fig. 3A, a significant decrease in NO level was detected in the stroke group compared with the control in both cerebral cortex and peripheral blood, while it increased dose-dependently in the CPT groups and demonstrated no significant difference compared with the LY294002 group.

CPT inhibits lipid peroxidation. SOD and MDA were measured to evaluate the oxidative damage caused by stroke (Fig. 3B and C). Similar results were obtained from both cerebral cortex and peripheral blood. Lower levels of SOD were detected in the stroke group, and this effect was attenuated in the CPT groups dose-dependently. Conversely, a higher content of MDA was measured in the stroke group, and it declined in the CPT groups in a dose-dependent manner. Both

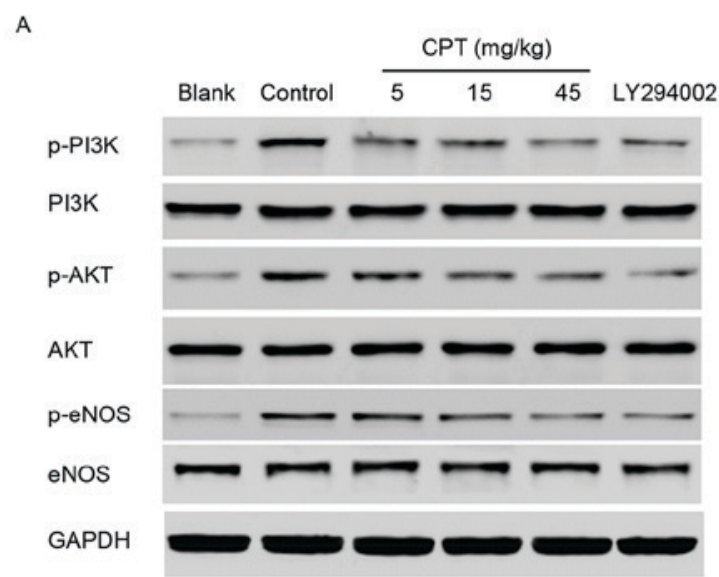

B

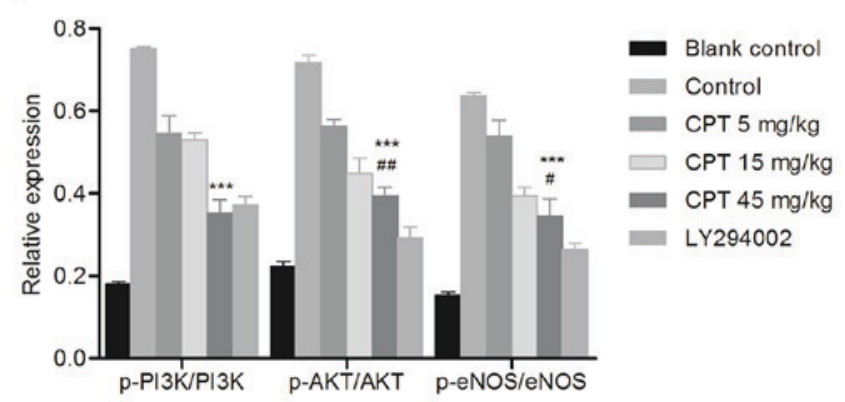

Figure 1. Phosphorylation of PI3K/AKT signaling pathway proteins, as measured by western blotting. (A) Representative western blot images and (B) quantification of PI3K, AKT and eNOS protein expression levels. GAPDH served as an internal control. Data are presented as the mean \pm standard deviation ( $\mathrm{n}=3$ independent experiments). ${ }^{* * *} \mathrm{P}<0.001$ vs. control group; ${ }^{\#} \mathrm{P}<0.05,{ }^{\#} \mathrm{P}<0.01$ vs. LY294002 group. AKT, protein kinase B; p, phosphorylated; eNOS, endothelial nitric oxide synthase; PI3K, phosphoinositide 3-kinase; CPT, cryptotanshinone.

MDA and SOD content in the control group were similar to the LY294002 group.

\section{Discussion}

PI3K has been implicated as a signaling enzyme activated by chemoattractant receptors $(14,15)$. A previous study reported that an agonist binding to the $\mathrm{C} 5 \mathrm{a}$ receptor can activate 
A

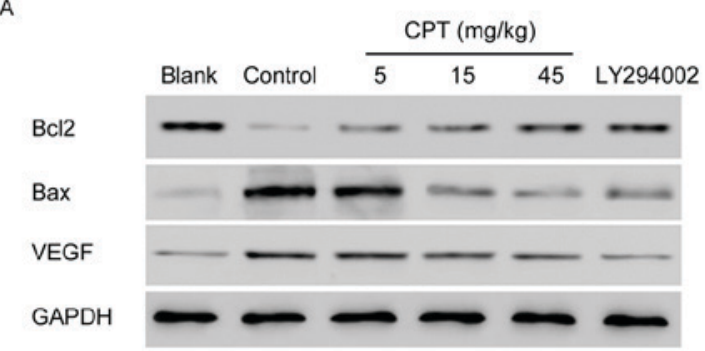

B

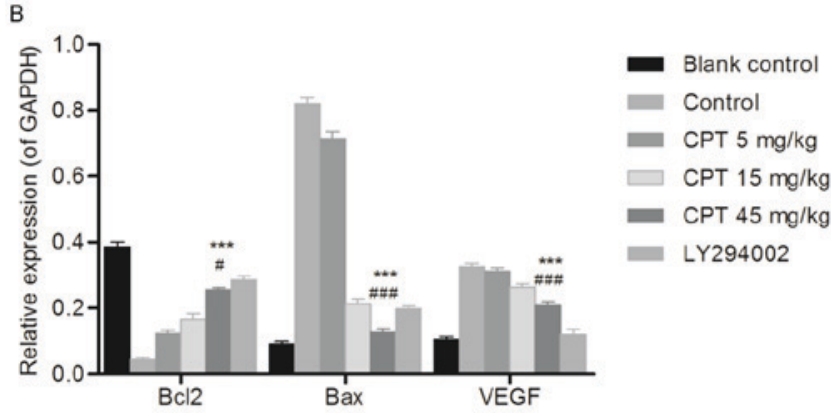

C

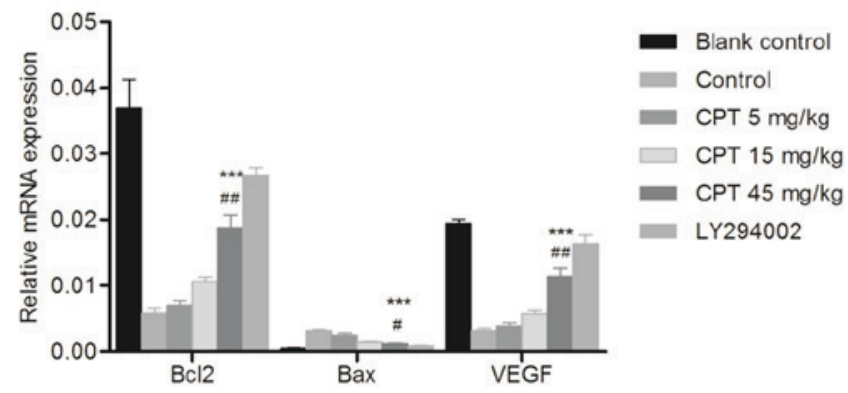

Figure 2. Expression level of phosphoinositide 3-kinase/protein kinase B signaling pathway downstream proteins, as measured by western blotting and reverse transcription-quantitative polymerase chain reaction. (A) Representative western blot images and (B) quantification of protein expression levels of Bcl-2, Bax and VEGF. GAPDH served as an internal control. (C) mRNA expression levels of Bcl-2, Bax and VEGF. Data are presented as the mean \pm standard deviation ( $\mathrm{n}=3$ independent experiments). ${ }^{* * * * *} \mathrm{P}<0.001$ vs. control group; ${ }^{\# \mathrm{P}}<0.05,{ }^{\# \#} \mathrm{P}<0.01,{ }^{\# \# \#} \mathrm{P}<0.001$ vs. LY294002 group. Bcl-2, B-cell lymphoma 2; Bax, Bcl-2-associated X protein; VEGF, vascular endothelial growth factor; CPT, cryptotanshinone.

multiple signaling proteins, including PI3K. The PI3K inhibitors wortmannin and LY294002 were demonstrated to inhibit chemotaxis in macrophages treated with chemoattractants (16). The activation of PI3K largely involves the substrates inside the plasma membrane. A variety of growth factors and signal transduction compounds, including fibroblast growth factor (FGF), VEGF, human growth factor, angiopoietin I (AngI) and insulin, can activate PI3K through the activation of receptor tyrosine kinase, resulting in phosphorylation $(17,18)$. The activation of PI3K leads to the generation of secondary messenger plasma membrane intrinsic protein 3 , which combines with signaling protein AKT and phosphoinositide dependent kinase-1 (PDK1). The combination induces the phosphorylation of Ser308 in AKT by PDK1, resulting in the activation of AKT. AKT is a major downstream effector of PI3K (19). AKT acts as an anti-apoptosis agent through the phosphorylation of downstream target proteins. AKT activates I $\kappa \mathrm{B}$ kinase, leading to the degradation of the $\mathrm{NF}-\kappa \mathrm{B}$ inhibitor $\mathrm{I} \kappa \mathrm{B}$, resulting in the activation of $\mathrm{NF}-\kappa \mathrm{B}$, so as to improve the

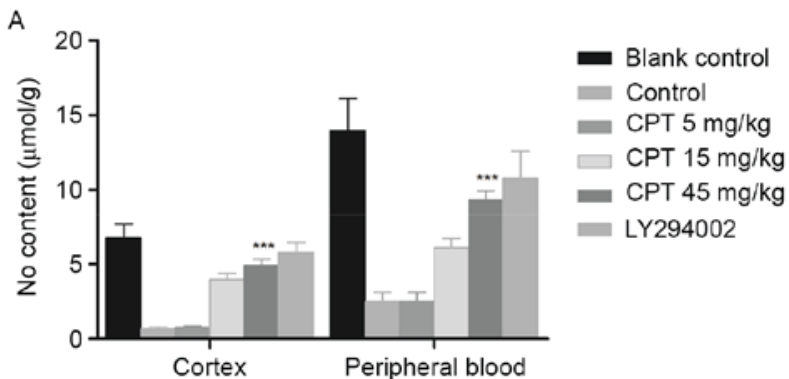

B
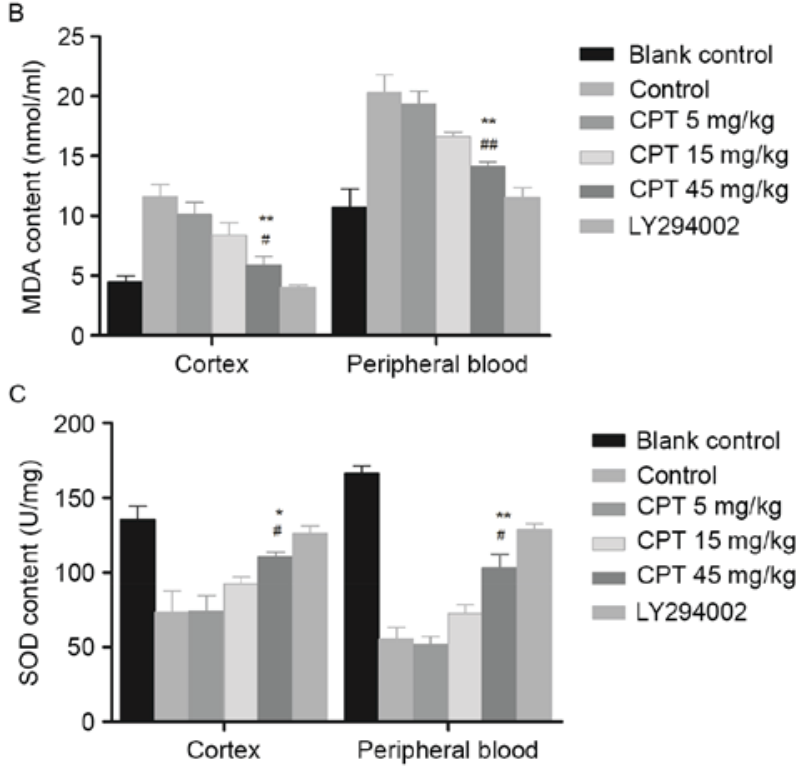

Figure 3. Content of NO, MDA and SOD in the cortex and peripheral blood. (A) NO, (B) MDA and (C) SOD content. Data are presented as the mean \pm standard deviation ( $\mathrm{n}=3$ independent experiments). ${ }^{*} \mathrm{P}<0.05,{ }^{* *} \mathrm{P}<0.01$, ${ }^{* * *} \mathrm{P}<0.001$ vs. control group; ${ }^{\#} \mathrm{P}<0.05,{ }^{\# \#} \mathrm{P}<0.01$ vs. LY294002 group. CPT, cryptotanshinone; NO, nitric oxide; MDA, malondialdehyde; SOD, superoxide dismutase.

survival of cells (12). The phosphorylation of Bcl-2-associated agonist of cell death, which belongs to Bcl-2 family, inhibits the combination to Bcl-extra large, resulting in the initiation of cell apoptosis. Additionally, AKT inhibits Caspase-9 so as to arrest the activation of apoptosis cascades. In the present study, reduced phosphorylation of PI3K in the CPT and LY294002 groups indicated the PI3K/AKT signaling pathway was suppressed. These findings suggested that CPT might inhibit the activation of PI3K as the PI3K inhibitor LY294002, leading to the declined AKT phosphorylation.

Furthermore, PI3Kg serves a critical role in the regulation of leukocyte functions, such as chemotaxis and superoxide production. Oxygen free radicals cause lipid peroxidation through attacking the polyunsaturated fatty acid to generate lipid peroxide, some of which can lead to cell metabolism and function disorder (20). MDA is a sensitive index of ROS-mediated lipid peroxidation which contributes to stroke damage, while SOD is an endogenous antioxidant enzyme developed to inhibit the production of ROS. Studies of MDA are generally combined with measuring SOD activity (21-23). The activity of SOD reflects the ability of remove oxygen free radicals, while the level of MDA indicates the damage of tissues and cells caused by free radicals. Previous studies demonstrated that the development of ROS serves an 
important role in tissue damage caused by stroke. Generated ROS in tissues experienced during ischemia and reperfusion is metabolized to prostaglandins and leukotrienes, resulting in the chemotaxis of leukocytes, and control of vascular endothelial cell function (24-26). In the present study, results obtained from biochemical tests demonstrated significantly increased MDA level and decreased SOD activity, demonstrating tissue damage caused by ROS-mediated lipid peroxidation during stroke. CPT dose-dependently attenuated SOD activity and MDA level, indicating the protective effect of CPT on cerebral tissue against lipid peroxidation.

NO, catalyzed by NOS synthase through L-arg and oxygen, is one of the hottest topics in the field of life science research in recent years (27). NO has a close association with cardiovascular, nervous, stomach, kidney and liver diseases (28). NO is a novel immune molecule and inflammatory mediator which mediates the pathological actions of cytokines including endotoxin, tumor necrosis factor and interleukin-6. eNOS serves a key role in the regulation of vascular function; it generates NO to expand blood vessels. On the other hand, it also generates superoxide, leading to vasoconstriction. It was reported that S-glutathionylation is involved in the oxidation-reduction switch of eNOS (29). It was observed to be increased in the blood vessels with hypertension, correlating with the damaged hemangiectasis. This type of oxidant stress occurs in heart attack, stroke, diabetes and cancer, suggesting the reset of this oxidation-reduction switch in order to restore the normal function of NOS may work as a potential therapy. In the present study, an increase in NO level was detected after the treatment of CPT at various concentrations, suggesting the hemangiectasis of blood vessels, indicating the protective effect of CPT on cerebral tissues against stroke.

In conclusion, CPT presented a protective effect against cerebral stroke through suppressing the PI3K/AKT-eNOS signaling pathway. Inhibited PI3K regulated the downstream effector protein AKT. Rats treated with CPT exhibited increased Bcl-2 and NO levels in both the cerebral cortex and peripheral blood, demonstrating the anti-apoptosis and blood vessel protection effect of CPT. Furthermore, increased SOD activity and declined MDA level demonstrated suppressed lipid peroxidation. The present study suggested the potential of CPT as a potential agent in the treatment of cerebral stroke.

\section{Acknowledgements}

The present study was supported by the Jinhua city, Zhejiang Province and Social Development to Focus on Subject (grant no. 2014-3-007) and The Public Welfare Technology Application Research (Experimental Animal) Project of Zhejiang Province (grant no. 2017F81SA700035).

\section{References}

1. Aries MJ, Elting JW, De Keyser J, Kremer BP and Vroomen PC: Cerebral autoregulation in stroke: A review of transcranial Doppler studies. Stroke 41: 2697-2704, 2010.

2. Stevenson SF, Doubal FN, Shuler K and Wardlaw JM: A systematic review of dynamic cerebral and peripheral endothelial function in lacunar stroke versus controls. Stroke 41: e434-e442, 2010.

3. Siniscalchi A, Gallelli L, Malferrari G, Pirritano D, Serra R, Santangelo E and De Sarro G: Cerebral stroke injury: The role of cytokines and brain inflammation. J Basic Clin Physiol Pharmacol 25: 131-137, 2014
4. Menon BK, Campbell BC, Levi C and Goyal M: Role of imaging in current acute ischemic stroke workflow for endovascular therapy. Stroke 46: 1453-1461, 2015.

5. Chen W, Lu Y, Chen G and Huang S: Molecular evidence of cryptotanshinone for treatment and prevention of human cancer. Anticancer Agents Med Chem 13: 979-987, 2013.

6. Kang BY, Chung SW, Kim SH, Ryu SY and Kim TS: Inhibition of interleukin-12 and interferon-gamma production in immune cells by tanshinones from Salvia miltiorrhiza. Immunopharmacology 49: 355-361, 2000.

7. Wang N, Luo HW, Niwa M and Ji J: A new platelet aggregation inhibitor from Salvia miltiorrhiza. Planta Med 55: 390-391, 1989.

8. Hur JM, Shim JS, Jung HJ and Kwon HJ: Cryptotanshinone but not tanshinone IIA inhibits angiogenesis in vitro. Exp Mol Med 37: 133-137, 2005.

9. Li S, Wang H, Hong L, Liu W, Huang F, Wang J, Wang P, Zhang X and Zhou J: Cryptotanshinone inhibits breast cancer cell growth by suppressing estrogen receptor signaling. Cancer Biol Ther 16: 176-184, 2015.

10. Don MJ, Liao JF, Lin LY and Chiou WF: Cryptotanshinone inhibits chemotactic migration in macrophages through negative regulation of the PI3K signaling pathway. Br J Pharmacol 151: 638-646, 2007.

11. Wu CY, Hsieh CY, Huang KE, Chang C and Kang HY: Cryptotanshinone down-regulates androgen receptor signaling by modulating lysine-specific demethylase 1 function. Int $\mathbf{J}$ Cancer 131: 1423-1434, 2012.

12. Kim EJ, Jung SN, Son KH, Kim SR, Ha TY, Park MG, Jo IG, Park JG, Choe W, Kim SS and Ha J: Antidiabetes and antiobesity effect of cryptotanshinone via activation of AMP-activated protein kinase. Mol Pharmacol 72: 62-72, 2007.

13. Livak KJ and Schmittgen TD: Analysis of relative gene expression data using real-time quantitative PCR and the 2(-Delta Delta C(T)) method. Methods 25: 402-408, 2001.

14. Stephens L, Ellson C and Hawkins P: Roles of PI3Ks in leukocyte chemotaxis and phagocytosis. Curr Opin Cell Biol 14: 203-213, 2002.

15. Hawkins PT, Anderson KE, Davidson K and Stephens LR: Signalling through Class I PI3Ks in mammalian cells. Biochem Soc Trans 34: 647-662, 2006.

16. Tsai HR, Yang LM, Tsai WJ and Chiou WF: Andrographolide acts through inhibition of ERK1/2 and Akt phosphorylation to suppress chemotactic migration. Eur J Pharmacol 498: 45-52, 2004.

17. Bai Y, Zhu X, Chao J, Zhang Y, Qian C, Li P, Liu D, Han B Zhao L, Zhang J, et al: Pericytes contribute to the disruption of the cerebral endothelial barrier via increasing VEGF expression: Implications for stroke. PLoS One 10: e0124362, 2015.

18. Koh SH and Lo EH: The role of the PI3K pathway in the regeneration of the damaged brain by neural stem cells after cerebral infarction. J Clin Neurol 11: 297-304, 2015.

19. Matsui T, Nagoshi T and Rosenzweig A: Akt and PI 3-kinase signaling in cardiomyocyte hypertrophy and survival. Cell Cycle 2: 220-223, 2003.

20. Cui HJ, Yang AL, Zhou HJ, Wang C, Luo JK, Lin Y, Zong YX and Tang T: Buyang huanwu decoction promotes angiogenesis via vascular endothelial growth factor receptor-2 activation through the PI3K/Akt pathway in a mouse model of intracerebral hemorrhage. BMC Complement Altern Med 15: 91, 2015.

21. Peralta C, Bulbena O, Xaus C, Prats N, Cutrin JC, Poli G, Gelpi E and Roselló-Catafau J: Ischemic preconditioning: A defense mechanism against the reactive oxygen species generated after hepatic ischemia reperfusion. Transplantation 73: 1203-1211, 2002.

22. Ozturk H, Gezici A and Ozturk H: The effect of celecoxib, a selective COX-2 inhibitor, on liver ischemia/reperfusion-induced oxidative stress in rats. Hepatol Res 34: 76-83, 2006.

23. Murakami K, Kondo T, Kawase M, Li Y, Sato S, Chen SF and Chan PH: Mitochondrial susceptibility to oxidative stress exacerbates cerebral infarction that follows permanent focal cerebral ischemia in mutant mice with manganese superoxide dismutase deficiency. J Neurosci 18: 205-213, 1998.

24. Kim ID, Sawicki E, Lee HK, Lee EH, Park HJ, Han PL, Kim KK, Choi H and Lee JK: Robust neuroprotective effects of intranasally delivered iNOS siRNA encapsulated in gelatin nanoparticles in the postischemic brain. Nanomedicine 12: 1219-1229, 2016.

25. Chandel NS, Trzyna WC, McClintock DS and Schumacker PT: Role of oxidants in NF-kappa B activation and TNF-alpha gene transcription induced by hypoxia and endotoxin. J Immunol 165: 1013-1021, 2000 
26. Du Y, Zhu Y, Teng X, Zhang K, Teng X and Li S: Toxicological effect of manganese on NF-kB/iNOS-COX-2 signaling pathway in chicken testes. Biol Trace Elem Res 168: 227-234, 2015.

27. Shiraki T, Aoyama T, Yokoyama C, Hayakawa Y, Tanaka T, Nishigaki K, Sawamura T and Minatoguchi S: LOX-1 plays an important role in ischemia-induced angiogenesis of limbs. PLoS One 9: e114542, 2014
28. Duran WN, Breslin JW and Sánchez FA: The NO cascade, eNOS location, and microvascular permeability. Cardiovasc Res 87: 254-261, 2010.

29. Kolluru GK, Siamwala JH and Chatterjee S: eNOS phosphorylation in health and disease. Biochimie 92: 1186-1198, 2010. 